GEORGE W. NORTHUP, D.O.

Editor in Chief

THOMAS W. ALLEN, D.O.

Scientific Editor

\section{Low-dose aspirin in the prevention of myocardial infarction}

The Food and Drug Administration recently approved the use of aspirin in the prevention of myocardial infarction in patients who have suffered previous myocardial infarction or unstable angina.

Results of six randomized, placebocontrolled, double-blind studies establish evidence of a 20 to 25 percent reduction in the incidence of cardiovascular mortality and nonfatal infarction with the use of aspirin. Furthermore, the results show that 83 of 100 patients treated with aspirin will have no additional cardiovascular events during the first year after discharge from the hospital.

Other studies have demonstrated beneficial effects of aspirin prophylaxis for reducing the incidence of stroke in patients who have experienced transient ischemic attacks. In patients suffering from unstable angina, there were reductions of 51 and 33 percent in the occurrence of deaths and infarction, respectively.

The author of this article suggests that a single tablet of $300 \mathrm{mg}$. of aspirin per day for prophylaxis would seem to be appropriate, because so far evidence about side effects from aspirin favors a low dose. Conversely, there has been no evidence that indicates a graded effect as dosages are increased. Other forms and doses of aspirin need to be tested for effectiveness in large, randomized, controlled trials.

Aspirin in combination with a beta blocker for therapy after infarction is not contraindicated. Together, their different mechanisms of action would likely produce an additive effect, according to the author.

Elwood, P.C.: Low-dose aspirin in the prevention of myocardial infarction. Practical Cardiology 13:53-60, Apr 87

\section{Vitamins as dietary supplements and therapeutic agents}

Healthy adults who are consuming nutritionally adequate diets do not need vitamins to supplement their food intake. Nutritional requirements are best met through a wide variety of foods, but because the eating habits of people in the United States have changed (more processed food, meals away from home, and between-meal snacks), vitamins delivered from the diet may be reduced. For individuals who are not obtaining adequate dietary vitamin intake, improvements in food selection and eating patterns are recommended. This report, from the Council on Scientific Affairs of the American Medical Association, emphasizes the importance of the proper use of vitamins, and the distinction between their functions as dietary supplements and therapeutic agents.

As a dietary supplement, vitamins are sometimes given to infants, to pregnant and lactating women, and, occasionally, to people with modified diets, regimens for weight reduction, and vegetarian diets that exclude all animal food products. As therapeutic agents, vitamins are indicated for the following conditions: deficiency diseases, malabsorption, prolonged illness, enteral and parenteral nutrition, alcoholism, burns, renal failure that requires dialysis, and genetic diseases. They may also be used to cause beneficial vitamin-nutrient and drug-vitamin interactions.

The health and nutrition of the public is best served by a scientific, sound basis for the use of vitamins as dietary supplements and therapy. Before any vitamins or vitamin mixtures are advocated for general use, their value should be established by convincing scientific research and evidence. Health care practitioners must provide patient education that stresses the importance of proper dietary food selection and its basis for good nutrition.

Council on Scientific Affairs: Vitamin preparations as dietary supplements and as therapeutic agents. JAMA 257:1929-36, 10 Apr 87

\section{Musculoskeletal injuries in competitive swimmers}

Increasing numbers of young athletes are engaging in competitive swimming each year. Because of the intensity of this sport, participants place themselves at an increased risk for musculoskeletal injuries. Shoulder pain, medial knee pain, and foot and ankle tendinitis are frequently reported overuse syndromes.

Shoulder pain, or "swimmer's shoulder," usually results from supraspinatus or biceps tendinitis. The disorder has been divided into $4 \mathrm{pha}$ ses, based on severity, and treatment is aimed at the particular phase of pain experienced. In the lower phases of shoulder pain, rehabilitation focuses on muscle strengthening. If the pain advances to phase 3 or 4 , resting the shoulder is usually indicated, until the pain diminishes to phase 1 or 2 , when rehabilitation can begin. 
Knee pain, or "breaststroker's knee," occurs most often in swimmers who use that stroke. Incorrect mechanics of the whip kick used during the breaststroke have been thought to be the cause of this syndrome. Tendinitis about the foot and ankle may result from the repetitive stretching extremes during ankle dorsiflexion and plantar flexion of swimmers trying to achieve an effective kick.

Prevention of overuse disorders in swimmers can be aided through proper training schedules, liberal warm-up and cool-down periods, stretching and strengthening programs, and proper stroke mechanics. Good communication and cooperation among swimmers, coaches, and physicians will ensure appropriate treatment and a rehabilitation program, designed to meet the individual needs of the athlete.

Johnson, J.E., Sim, F.H., and Scott, S.G.: Musculoskeletal injuries in competitive swimmers. Mayo Clin Proc 62:289-304, Apr 87

\section{Managing the elderly patient with hypertension and pulmonary disease}

Management of the elderly patient who has both hypertension and pulmonary disease can be a challenge for clinicians. Critical to this management is the selection of therapeutic agents that will not interfere or aggravate either concurrent disorder. The authors of this article describe common antihypertensive treatments and their advantages and disadvantages for patients with pulmonary disease.

Diuretics, central-acting alpha ${ }_{2}$ agonists, direct vasodilators, and alpha $_{1}$-antagonists have proved efficacy, but their possible side effects (mucosal drying and electrolyte imbalance, oversedation and depressed respiration, increased risk of myocarcial ischemia, and salt retention) may limit their usefulness in treating elderly patients. Beta-adrenergic blockers and peripheral adrenergic inhibitors are contraindicated because of their adverse pulmonary effects. Calcium-channel blockers and ACE-inhibitors have shown good response in elderly pa- tients, particularly those hypertensive patients with chronic obstructive pulmonary disease.

Because of the range of therapeutic agents available today, physicans are able to safely and effectively manage both the hypertension and pulmonary disease of elderly patients.

Krane, N.K., and Wallin, J.D.: Managing the elderly patient with both hypertension and pulmonary disease. Geriatrics 42:45-52, Apr 87

\section{AIDS knowledge and beliefs among adolescents}

Twenty-one percent of the reported cases of acquired immunodeficiency syndrome (AIDS) to the Centers for Disease Control involve individuals in the 20- to 29-year age group. Given the sexual behavior and drug use among adolescents, and the disease's latency period of 1 to more than 4 years, adolescents are at high risk for exposure to the AIDS virus. A July 1985 survey among San Francisco teenagers showed that although most knew that sexual intercourse and sharing intravenous drug needles were modes of contraction, only 60 percent knew ways of avoiding transmission, and more than onethird believed that handshaking could spread AIDS. These findings showed that despite mass media focus on AIDS cases, reports of heterosexual transmission, and the call for public education, many adolescents were misinformed about the disease. The present study was conducted between August and October 1986 among Massachusetts teenagers aged 16 to 19 years.

The 829 teenagers who agreed to participate in the telephone survey were selected by random-digit dial sampling. Questions were asked to assess the adolescents' knowledge about how AIDS is transmitted, who is at high risk for exposure, ways of avoiding exposure, and appropriate behavior with people who have AIDS.

Almost all of the respondents knew that AIDS can be transmitted through homosexual intercourse between two men; however, 8 percent were unaware about possible heterosexual transmission. Only 7 percent thought or did not know that blood transmission is a means of spreading the virus, 60 percent thought that giving blood could transmit AIDS, and the majority were unclear about transmission through other body fluids. Almost one-fourth believed that persons with AIDS should not be allowed to attend school. Of those who were sexually active, just 15 percent had changed their sexual behavior because of concern about AIDS (only 20 percent of this subgroup were found to be using effective prevention methods. Eight percent of the drug users did not know that AIDS could be spread by injecting drugs, and of those who knew someone with AIDS, 11 percent believed the person to be contagious.

These results indicate that confusion and misconception about AIDS still exist even among groups at high risk for exposure. Knowledge about AIDS is greater than in the 1985 survey, but more teenagers are not worried about contracting it now, as compared to 1985 . School intervention programs and additional surveys are suggested to provide further education and to monitor adolescents' knowledge and beliefs about AIDS.

Strunin, L., and Hingson, R.: Acquired immunodeficiency syndrome and adolescents: Knowledge, beliefs, attitudes, and behaviors. Pediatrics 79:825-8, May 87

\section{Evaluation of commercial rapid bacteriuria screening procedures}

The quantitative urine culture is a widely accepted and utilized procedure for the diagnosis of urinary tract infection in female patients. During the last few years, a number of commercial bacteriuria screening tests have been marketed and evaluated in comparison with the standard culture method for their ability to detect a minimum of $10^{4}$ colony forming units of microorganisms. This study was conducted to evaluate performance characteristics-sensitivity, specificity, and predictive value of positive and negative testsof two commercially available rapid bacteriuria screening methods on obstetric and nonpregnant female populations. 


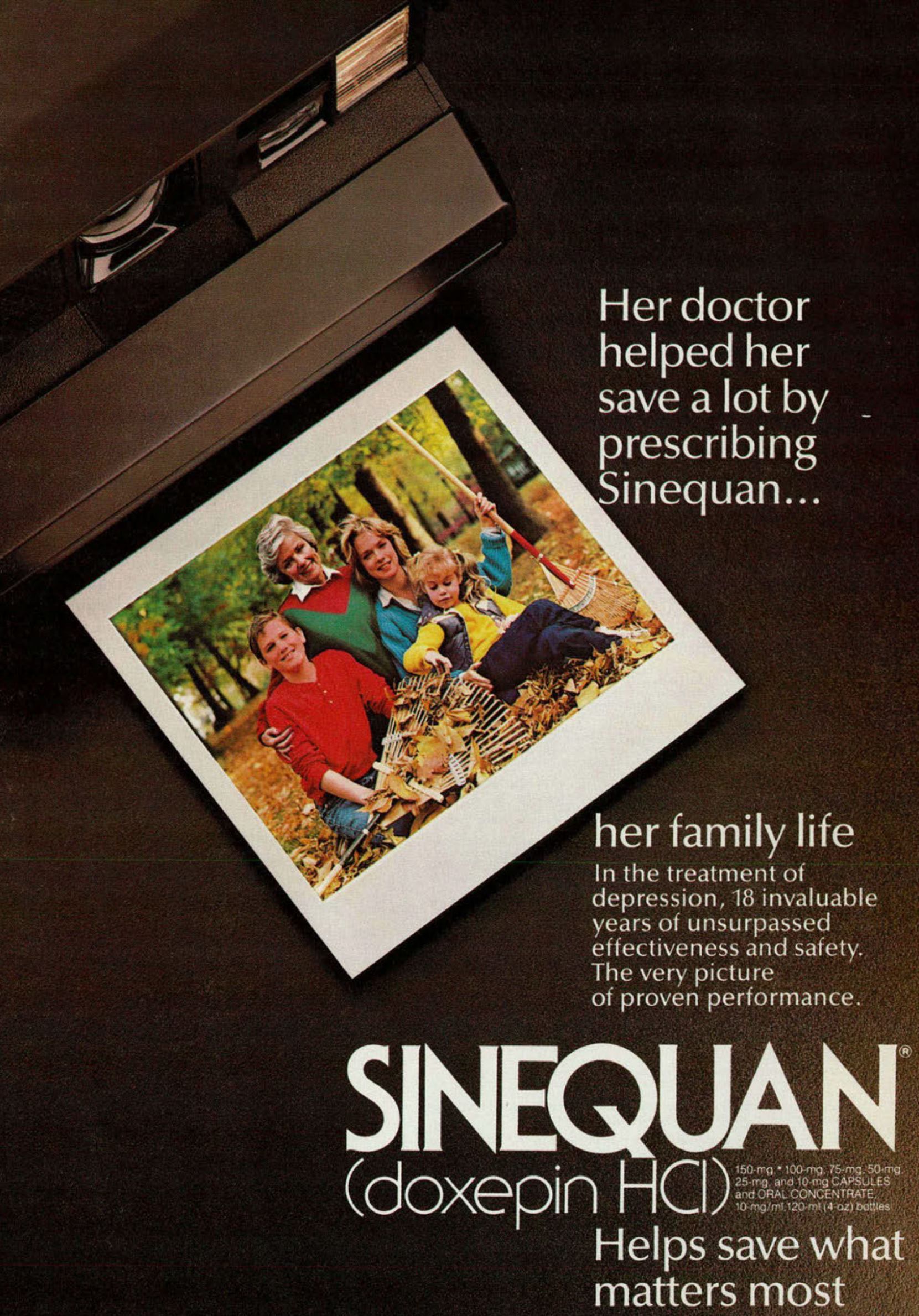




\section{BRIEF SUMMARY}

SINEQUAN* (doxepin $\mathrm{HCl}$ ) Capsules/0ral Concentrate

Indications. SINEQUAN is recommended for the treatment tot:

Psychoneurotic patients with depression and/or anxiety.

2. Depression and/or anxiety associated with alcoholism (not to be taken concomitantly with alcohol).

3. Depression and/or anxiety associated with organic disease (the possibility of drug interaction should be considered if the patient is receiving other drugs concomitantly)

4. Psychotic depressive disorders with associated anxiety including involutional depression and manic-depressive disorders.

The target symptoms of psychoneurosis that respond particularly well to SINEQUAN include anxiety. tension, depression, somatic symptoms and concerns, sleep disturbances, guilt, lack of energy, fear. apprehension and worry

Clinical experience has shown that SINEQUAN is safe and well tolerated even in the elderly patient. Owing to lack of clinical experience in the pediatric population, SINEQUAN is not recommended for use in children under 12 years of age

Contraindications. SINEQUAN is contraindicated in individuals who have shown hypersensitivity to the drug. Possibility of cross sensitivity with other dibenzoxepines should be kept in mind

SINEQUAN is contraindicated in patients with glaucoma or a tendency to urinary retention. These disorders should be ruled out, particularly in oider patients.

Warnings. The once-a-day dosage regimen of SINEQUAN in patients with intercurrent iliness or patients taking other medications should be carefully adjusted. This is especially important in patients receiving other medications with anticholinergic effects.

Usage in Geriatrics: The use of SINEQUAN on a Once-a-day dosage regimen in geriatric patients should be adjusted carefulily based on the patient's condition.

Usage in Pregnancy: Reproduction studies have been performed in rats, rabbits. monkeys and dogs and there was no evidence of harm to the animal fetus. The relevance to humans is not known. Since there is no experience in pregnant wornen who have received this drug. satety in pregnancy has not been established. There are no data with respect to the secretion of the drug in human milk and its effect on the nursing infant.

Usage in Children: The use of SINEQUAN in children under 12 years of age is not recommended because safe conditions for its use have not been established

MAO Inhibitors: Serious side effects and even death have been reported following the concomitant use of certain drugs with MAO inhibitors. Therefore. MAO inhibitors should be discontinued at least two weeks prior to the cautious initiation of therapy with SINEQUAN. The exact length of time may vary and is dependent upon the particular MAO inhibitor being used, the length of time it has been administered. and the dosage involved

Usage with Alcohol: It should be borne in mind that alcohol ingestion may increase the danger inherent in any intentional or unintentional SINEQUAN overdosage. This is especially important in patients who may use alcohol excessively.

Precautions. Since drowsiness may occur with the use of this drug. patients should be warned of the possibility and cautioned against driving a car or operating dangerous machinery while taking the drug. Patients should also be cautioned that their response to aiconol may be potentiated.

Since suicide is an inherent risk in any depressed patient and may remain so until significant improvement has occurred, patients should be closely supervised during the early course of therapy. Prescriptions should be written for the smailest feasible amount.

Should increased symptoms of psychosis or shift to manic symptomatology occur, it may be ecessary to reduce dosage or add a major tranquilizer to the dosage regimen.

Adverse Reactions. NOTE: Some of the adverse reactions noted below have not been specifically reported with SINEQUAN use. However, due to the close pharmacological similarities among the tricyclics, the reactions should be considered when prescribing SINEQUAN

Anticholinergic Effects: Dry mouth, blurred vision, constipation, and urinary retention have been reported. If they do not subside with continued therapy, or become severe, it may be necessary to reduce the dosage.

Central Nervous System Effects: Drowsiness is the most commonly noticed side effect. This tends to disappear as therapy is continued. Other infrequently reported CNS side effects are confusion, disorientation, hallucinations, numbness, paresthesias, ataxia, and extrapyramidal symptoms and seizures. Cardiovascular: Cardiovascular effects including hypotension and tachycardia have been reported occasionally.

Allergic: Skin rash, edema, photosensitization, and pruritus have occasionally occurred. Hematologic. Eosinophilia has been reported in a few patients. There have been occasional reports of Done marrow depression manifesting as agranuiocytosis, leukopenia, thrombocytopenia, and purpura. Gastrointestinal: Nausea, vomiting, indigestion, taste disturbances, diarrhea, anorexia, and aphhous stomatitis have been reported. (See anticholinergic effects.)

tia in males, enlargement of breasts and galactorrhea in the female, raising or lowering of blood sugar levels, and syndrome of inappropriate antidiuretic hormone have been reported with tricyclic administration.

Other: Dizziness, tinnitus, weight gain, sweating. chills, fatigue, weakness, flushing, jaundice,

Withdrawa/ Symptoms: The possibility of development of withdrawal symptoms upon abrupt cessa. tion of treatment after prolonged SINEQUAN (doxepin $\mathrm{HCl}$ ) administration should be borne in mind These are not indicative of addiction and gradual withdrawal of medication should not cause these symptoms.

列 dose of $75 \mathrm{mg}$ is recommended. Dosage may subsequently be increased or decreased at appropriate In more severely ill patients higher doses may be required with subsequent gradual increase to $300 \mathrm{mg} /$ day if necessary. Additional therapeutic effect is rarely to be obtained by exceeding a dose of $300 \mathrm{mg} / \mathrm{day}$.

In patients with very mild symptomatology or emotional symptoms accompanying organic disease. ower doses may suffice. Some of these patients have been controlled on doses as $10 \mathrm{w}$ as $25-50 \mathrm{mg} / \mathrm{day}$ The total daily dosage of SINEQUAN may be given on a divided or once-a-day dosage schedule. If the once-a-day schedule is employed the maximum recommended dose is $150 \mathrm{mg} /$ day. This dose may be given at bedtime. The $150 \mathrm{mg}$ capsule strength is intended for maintenance therapy only and is not recommended for initiation of treatment.

Anti-anxiety effect is apparent before the antidepressant effect. Optimal antidepressant effect may not be evident for two to three weeks.

Overdosage.

A. Signs and Symptoms

Mild: Drowsiness, stupor, blurred vision, excessive dryness of mouth

2. Severe: Respiratory depression, hypotension, coma, convulsions, cardiac arrhythmias and tachycardias

Also: urinary retention (bladder atony), decreased gastrointestinal motility (paralytic ileus), hyperthermia (or hypothermia), hypertension, dilated pupils, hyperactive reflexes.

B. Management and Treatment

Mild: Observation and supportive therapy is all that is usually necessary

2. Severe: Medical management of severe SINEQUAN overdosage consists of aggressive supportive herapy. If the patient is conscious, gastric lavage, with appropriate precautions to prevent pulmonary aspiration, should be performed even though SINEQUAN is rapidly absorbed. The use of activated charcoal has been recommended, as has been continuous gastric lavage with saline for 24 hours of more. An adequate airway should be established in comatose patients and assisted ventilation used if necessary. EKG monitoring may be required for several days, since relapse after apparent recovery has been reported. Arrhythmias should be treated with the appropriate antiarrhythmic agent. It has been reported that many of the cardiovascular and CNS symptoms of tricyclic antidepressant poisoning in adults may be reversed by the slow intravenous administration of $1 \mathrm{mg}$ to $3 \mathrm{mg}$ of physostigmine salicylate. Because physostigmine is rapidly metabolized, the dosage should be repeated as required. Convulsions may respond to standard anticonvulsant therapy, however, barbiturates may potentiate any respiratory depression. Dialysis and forced diuresis generally are not of value in the management of overdosage due to high tissue and protein binding of SINEQUAN

More detailed professional information available on request.
Urine samples were obtained from 694 obstetric and 143 nonpregnant women. The following procedures were performed for all 837 specimens: routine quantitative culture, and commercial procedures Chemstrip LN (Biodynamics, Indianapolis, Indiana) and Bac-T-Screen, (Marion Laboratories, Kansas City, Missouri).

In obstetric patients, of 56 significant bacteriurias, LN screen correctly identified 39 , and Bac-TScreen, 54. Of 638 nonsignificant culture findings, LN identified 532, and Bac-T-Screen, 357. In nonpregnant patients, of 32 significant bacteriurias, LN screen identified 23, and Bac-T-Screen, 27. Of 111 nonsignificant culture findings, $\mathrm{LN}$ screen correctly identified 84 , and Bac-TScreen, 73 .

Commercial screening tests for significant bacteriuria should be able to identify large quantities of negative samples and provide maximum diagnostic sensitivity. In addition, the tests should be cost effective, quick, and clinically useful. The Chemstrip LN method was found unacceptable because of its low sensitivity (70 percent) for significant bacteriuria. Bac-T-Screen showed excellent sensitivity ( 96 percent); however, because of a high rate of false-positive findings ( 281 in obstetric patients, which included 171 contaminated specimens, and 38 in nonpregnant patients, including 13 contaminated specimens), and the expense of the test, its routine use is limited. The authors conclude that routine quantitative culture, where readily available, continues to be the most cost-effective detection method for significant bacteriuria in women.

McNeely, S.G., Baselski, V.S., and Ryan, G.M.: An evaluation of two rapid bacteriuria screening procedures. Obstet Gynecol 69:550-3, Apr 87

\section{Sources of conflict in the medical marriage}

Despite the fact that many physicians and physicians' spouses report unhappiness in their marriages, they tend to stay with their mates rather than divorce and seek new partners. Underlying contributing 
factors to unhappiness and conflict in the medical marriage are not very clear. The literature has cited the major source of conflict to be the long hours worked by the physician which leave little time for spousal and family interaction. Despite this explanation's popularity, studies have reported no significant correlation between long work hours and either divorce or marital unhappiness. The authors of this article report their findings of a study of a group of medical marriages, which was conducted to better understand sources of the discontent.

A group of 240 couples who participated in a 5-day continuing education workshop were sent questionnaires. Information was obtained from each spouse concerning demographics, working patterns, psychologic health, and gratification and sources of conflict in the marriage.

Both groups reported that not having enough time for family, fun, and self was the number-one source of marital conflict. Discrepancies about sources of conflict existed in two areas: (1) in the perception of amount of time spent at work and away from home; and (2) in the lack of intimacy. Physicians saw the amount of time away from home, while the spouses saw a lack of intimacy as the greater source of discord.

The data were consistent with the idea that less marital counseling is needed among couples who communicate more. The results also suggest that the complaint of not having enough time for the spouse is more of an excuse than an explanation for marital unhappiness. The complaint serves as a means of placing blame on an external factor for problems within the marriage. No relationship was found between long working hours and discord in the medical marriage. Instead, differences in communication styles, ways of expressing feelings, needs for intimacy, and perceptions of marital problems may contribute to the sources of conflict in the relationship.

Gabbard, G.O., Menninger, R.W., and Coyne, L. Sources of conflict in the medical marriage. Am J Psychiatry 144:567-72, May 87

\section{Menopause and risk of coronary heart disease}

Recent studies have shown a possible association between postmenopausal estrogen-replacement therapy and a decrease in the risk of coronary heart disease. In the present study, menopausal status was examined to see whether its possible influence on the risk of coronary heart disease and myocardial infarction might be altered by postmenopausal estrogenreplacement therapy.

In 1976, 121,700 U.S. women aged $30-55$ years were recruited for this study and were mailed questionnaires. Of these, 116,258 , who had no diagnosis of coronary heart disease and who were premenopausal or had a known type of menopause, formed the study population. Follow-up questionnaires were mailed in 1978 , 1980, and 1982.

The study revealed that after controlling for age and cigarette use, the women who had had natural menopauses, with or without estrogen replacement, faced no appreciable increase in risk for coronary heart disease compared to the premenopausal women. Subsequent analyses on postmenopausal women showed that those who had never used estrogens after bilateral oophorectomy had an increased risk of coronary heart disease of 2.2 , as compared to premenopausal women. Among those who did use estrogens after this type of surgical menopause, no increased risk of coronary heart disease was evident. Although chance, bias, and residual confounding may explain some of these findings, the data obtained from this study support the hypoth- esis that risk of coronary heart disease is increased after bilateral oophorectomy, probably because of the loss of endogenous estrogens. Further investigation is needed to replicate these results and to study the effects of menopausal duration on coronary heart disease.

Colditz, G.A., et al.: Menopause and the risk of coronary heart disease in women. $\mathrm{N}$ Engl $\mathrm{J}$ Med 316:1105-10, 30 Apr 87

\section{Do adhesions cause pain?}

Operative trauma, infection, hemorrhage, and radiation may cause peritoneal damage and adhesions between the peritoneal surfaces can result. These can vary in form from a single band to a tough, encircling cocoon. Although it is generally accepted among abdominal surgeons that adhesions can cause laparotomy difficulties and strangulation, controversy exists as to whether intraabdominal or pelvic pain can result from adhesions.

The author of this article believes that there are no clear answers regarding the possible painful symptoms of adhesions and the division and prevention of further adhesions. Only opinions are available, with his own being that it is "a poorly substantiated myth that adhesions cause abdominal or pelvic pain." Many anecdotes exist about adhesions being divided and the subsequent relief of patients' specific abdominal symptoms; however, a placebo effect should not be overlooked.

Sometimes adhesions must be divided, in the author's opinion, particularly when reoperation requires total gut assessment, or in the case of single, tough, bandlike adhesions that could result in strangulation.

Alexander-Williams, J.: Do adhesions cause pain? Br Med J 294:659-60, 14 Mar 87 